\title{
Childhood longitudinal melanonychia: case series from Poland
}

\author{
Michał Sobjanek ${ }^{1}$, Martyna Sławińska ${ }^{1}$, Alicja Romaszkiewicz ${ }^{1}$, Wojciech Biernat ${ }^{2}$, Rafał Pęksa², Roman J. Nowicki ${ }^{1}$
}

1Department of Dermatology, Venereology and Allergology, Medical University of Gdańsk, Gdańsk, Poland 2Department of Pathomorphology, Medical University of Gdańsk, Gdańsk, Poland

Adv Dermatol Allergol 2020; XXXVII (2): 195-201

DOI: https://doi.org/10.5114/ada.2019.87706

\begin{abstract}
Introduction: Longitudinal melanonychia (LM) is characterized by a tan, brown or black longitudinal streak within nail plate caused by the presence of melanin. LM is relatively common in dark-skinned population, infrequent in Caucasian population, and rare in children.

Aim: We report epidemiological, clinicopathological and dermoscopic analysis of 8 cases of childhood LM from Poland, which is the largest series in the Central and Eastern European population.

Material and methods: Three hundred and forty-eight patients presenting with various nail pigmentation (in 2010-2016) were analysed. 72 cases of LM have been identified, including 8 cases of childhood LM ( $<16$ years of age), which were included in further analysis.

Results: Seven patients were boys and one girl, with mean age of 9 years (range: 6-13). More than a half $(n=5)$ presented skin phototype II. The most common location of melanonychia was the first left fingernail. Dermoscopy revealed heterogeneity of longitudinal lines colour in 5 cases. The irregularity of longitudinal line thickness in 5 cases and irregularity of parallelism in 5 cases was observed. Histopathological evaluation was performed in 4 patients, in 3 cases it revealed the presence of nail matrix nevus, in one case the presence of melanocytic proliferation of the lentiginous pattern along the dermoepidermal junction.

Conclusions: Despite the fact that melanoma was not recognised in any case, such a possibility should always be considered as the cause of LM, even in the paediatric population. Dermoscopy seems to be useful in patient follow-up and management.
\end{abstract}

Key words: longitudinal melanonychia, nail apparatus melanoma, children, dermoscopy.

\section{Introduction}

Longitudinal melanonychia (LM) also known as melanonychia striata is defined as a grey to black pigmentation of the nail plate due to the presence of melanin caused by hyperplasia or activation of nail matrix melanocytes [1]. The most important clinical aspect of LM is the association with the nail apparatus melanoma (NAM). It is estimated that approximately $76 \%$ of cases of this neoplasm presents initially as LM [1, 2]. NAM represents from $0.7 \%$ to $3.4 \%$ of all diagnosed melanomas in the Caucasian population. Rarity of this entity and non-specific clinical presentation contribute to the delay of the treatment due to inaccurate initial diagnosis. This determines worse prognosis in comparison to cutaneous melanoma [3].

The incidence of $L M$ depends on genetically determined differences in the number and activity of mela- nocytes localized in nail apparatus. LM is relatively common in Afro-American, Japanese and Latino population. According to literature data, the occurrence of $L M$ in Caucasian population does not exceed $1 \%$. LM is rarely observed in children [1-5]. In the Polish literature this medical issue has not been reported yet.

\section{Aim}

To present the largest series of childhood LM in the Central and Eastern European population.

\section{Material and methods}

We analysed 348 patients who have been diagnosed with various nail pigmentation in 2010-2016. In adults, 64 cases of LM have been identified (including 3 cases

Address for correspondence: Alicja Romaszkiewicz MD, Department of Dermatology Venereology and Allergology, Medical University of Gdańsk, 17 Smoluchowskiego St, 80-214 Gdansk, Poland, phone: +48 5858440 10, e-mail: romaszkiewicz@gumed.edu.pl Received: 18.03.2018, accepted: 1.10.2018. 


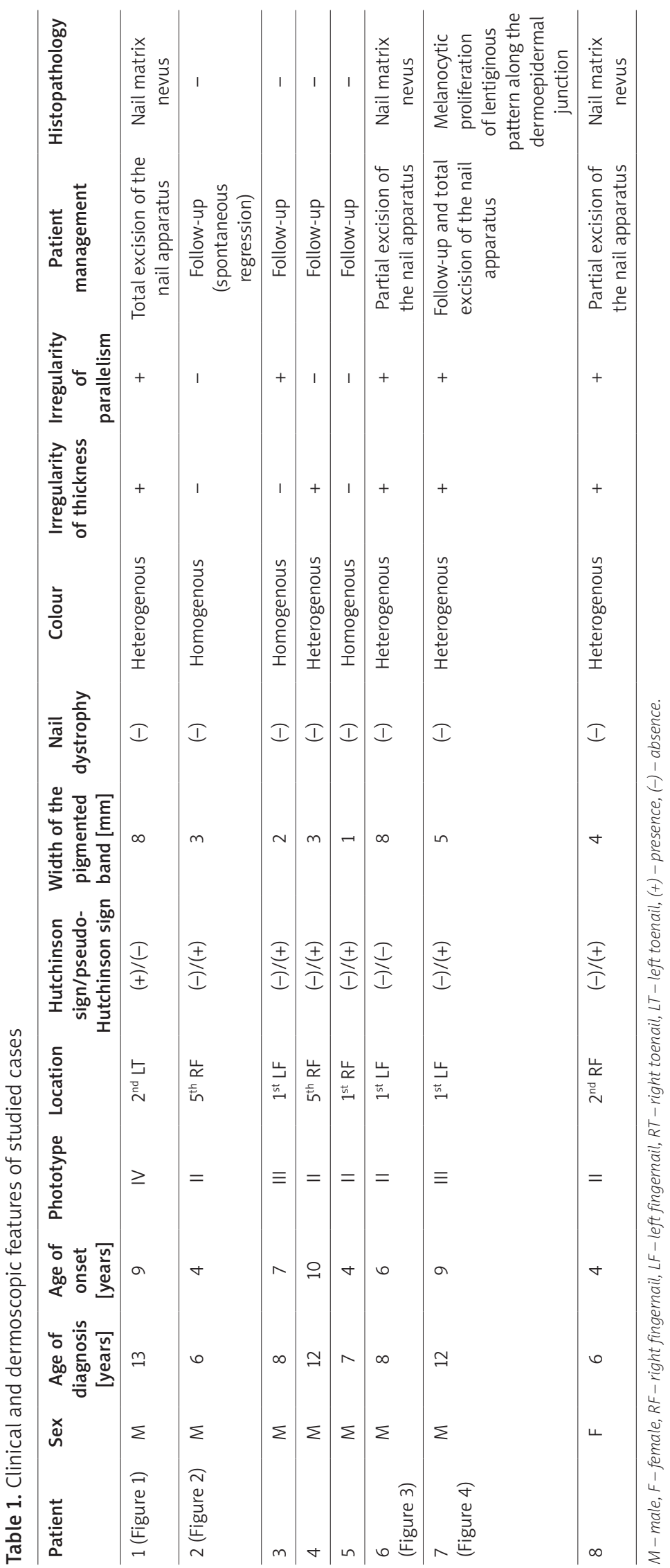

of NAM; $4.7 \%$ ), whereas 8 cases of LM have been identified in children ( $<16$ years of age). The latter were included in the further detailed analysis. Final diagnosis was established according to clinical, dermoscopic (onychoscopic) and histopathological evaluation.

\section{Results}

Clinicopathological and dermoscopic features of the analysed cases are summarized in Table 1 (Figures 1-4). Mean age at diagnosis was 9 years (range: $6-13$ ), whereas the mean age of LM onset was 6.6 years (range: $4-10$ ). There was no personal or family history of melanoma in the studied group. All children were otherwise healthy. No preceding trauma, history of medications intake or pigmentationrelated disorders were reported.

The most common location of melanonychia was the left first fingernail. Mean width of the pigmented band was $4.3 \mathrm{~mm}$. In 2 cases, pigmentation involved $>50 \%$ of the nail plate; in 3 cases, $>30 \%$ of the nail plate. The Hutchinson sign was present in one case, and pseudoHutchinson sign in 6 cases. Nail dystrophy has not been observed. Dermoscopically, the colour heterogeneity of longitudinal lines within a band was present in 5 cases; the irregularity of longitudinal lines thickness was present in 5 cases and irregularity of parallelism in 5 cases.

In 3 patients, the histopathological examination was performed after initial evaluation. In 1 patient (patient 1, Figure 1 A), after considering the doubtful character of the lesion, mother's anxiety and size of the lesion, a decision of total excision of the nail apparatus was made. The procedure was performed in conduction anaesthesia. The nail apparatus was excised in one piece, the wound was covered with full-thickness skin graft taken from the inguinal area. The postsurgical period was uncomplicated. Further aesthetic results were satisfying (Figure 1 B). Histopathological examination revealed the presence of nail matrix nevus.

In 5 patients, the regular follow-up was recommended. In patient 2, spontaneous regression of LM was observed. Clinical examination at the first visit revealed $3 \mathrm{~mm}$ wide brown longitudinal streak of the right fifth fingernail; after 6 months, previously observed pigmentation was absent (Figures $2 \mathrm{~A}, \mathrm{~B}$ ).

In patient 7 , after 13-month follow-up, based on the worrying change in clinical and dermoscopic presentation (Figures 4 A, B), a decision of total excision of the nail plate was 

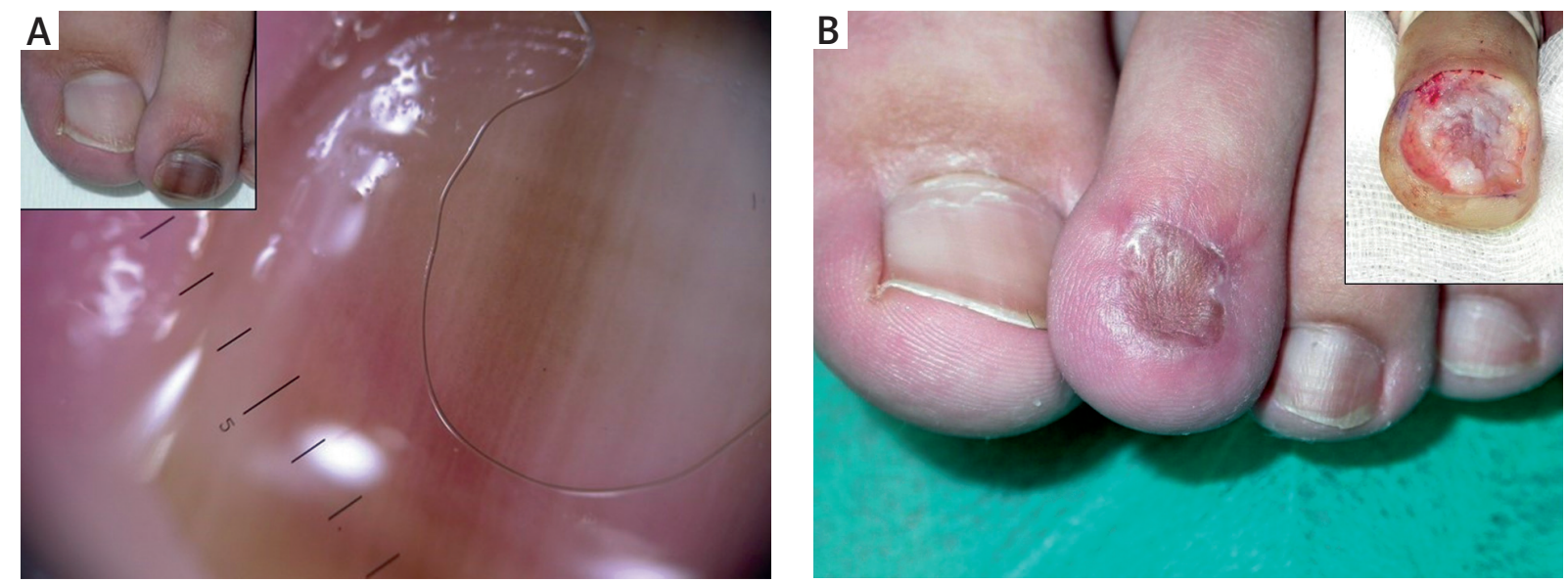

Figure 1. Patient 1. A - LM involving most of the second toenail plate with the presence of Hutchinson sign (clinical and dermoscopic presentation). Heterogeneity in colour and irregularity of thickness and parallelism of the longitudinal lines. B - Clinical presentation after wide local excision of the nail apparatus and well-healed toenail after skin graft
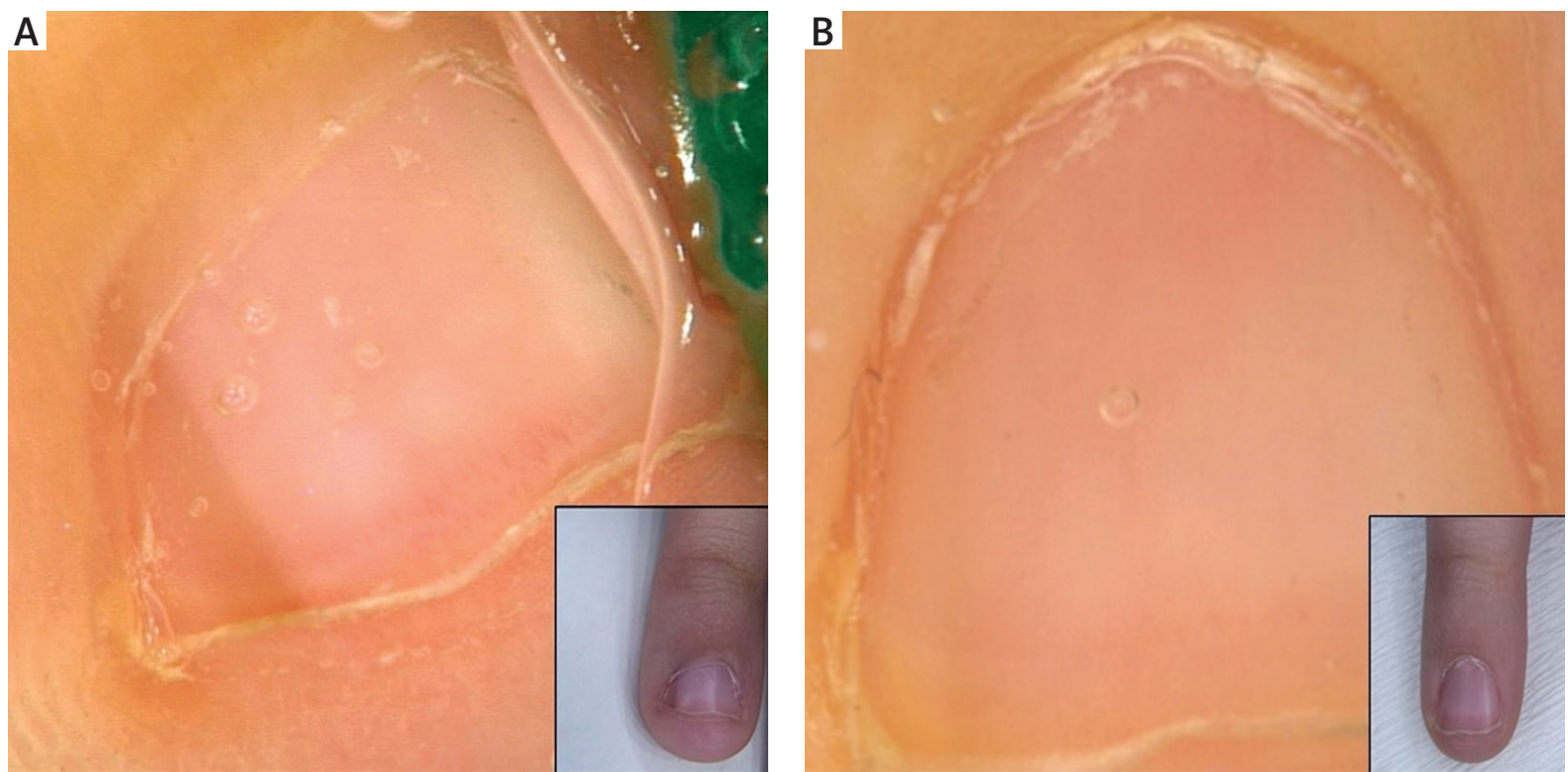

Figure 2. Patient 2. A - LM of the fifth right fingernail (clinical and dermoscopic presentation). Longitudinal, homogenous in colour, regularly distributed pigmented bands. B - Spontaneous regression of LM after 6 months (clinical and dermoscopic presentation)

made. Histopathological examination revealed the presence of melanocytic proliferation of the lentiginous pattern along the dermoepidermal junction (Figures $5 \mathrm{~A}, \mathrm{~B}$ ).

\section{Discussion}

LM incidence in the Caucasian population is estimated to be approximately 1\% [3]. Nevertheless, authors' observations indicate a much lower frequency of this lesion. The overstatement of the data may result from inconsistency of nomenclature. Some researchers classify lesions with non-melanocytic origin (e.g. subungual haemorrhage) into a group of melanonychia $[5,6]$. Inde-

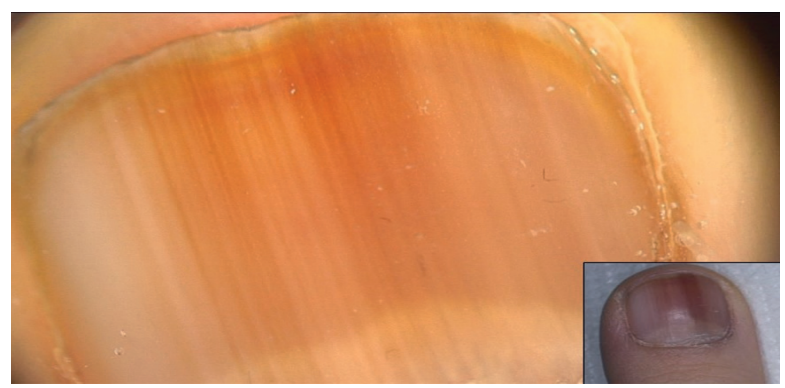

Figure 3. Patient 6. LM of the left thumb (clinical and dermoscopic presentation). Heterogeneity in colour and irregularity of thickness and parallelism of the longitudinal lines 

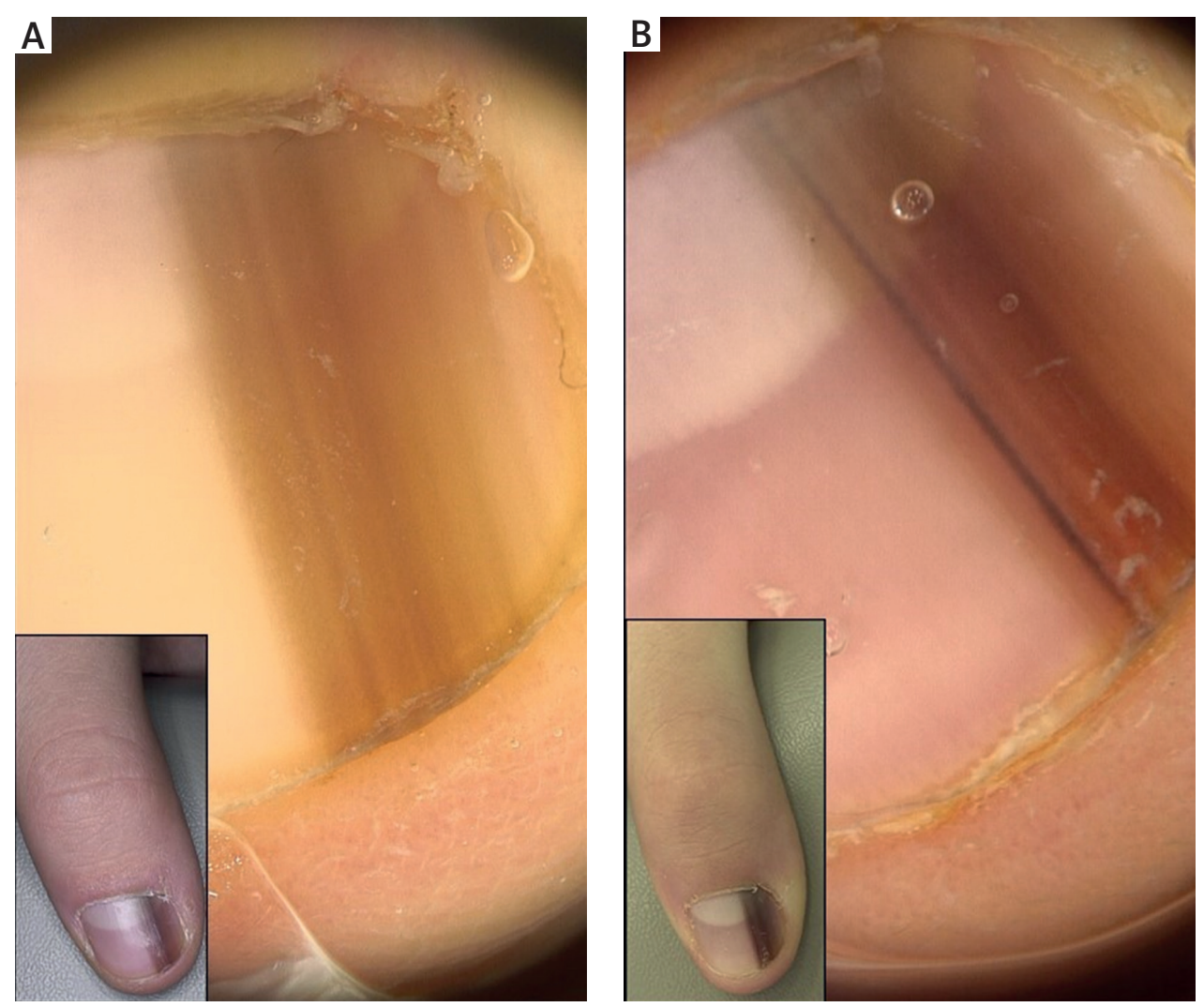

Figure 4. Patient 7. A - LM of the left thumb (initial clinical and dermoscopic presentation). Moderate heterogeneity in colour and irregularity of thickness and parallelism of the longitudinal lines. B - LM of the left thumb - a significant change in clinical and dermoscopic presentation after 13-month follow-up. Remarkable heterogeneity in colour and irregularity of thickness and parallelism of the longitudinal lines
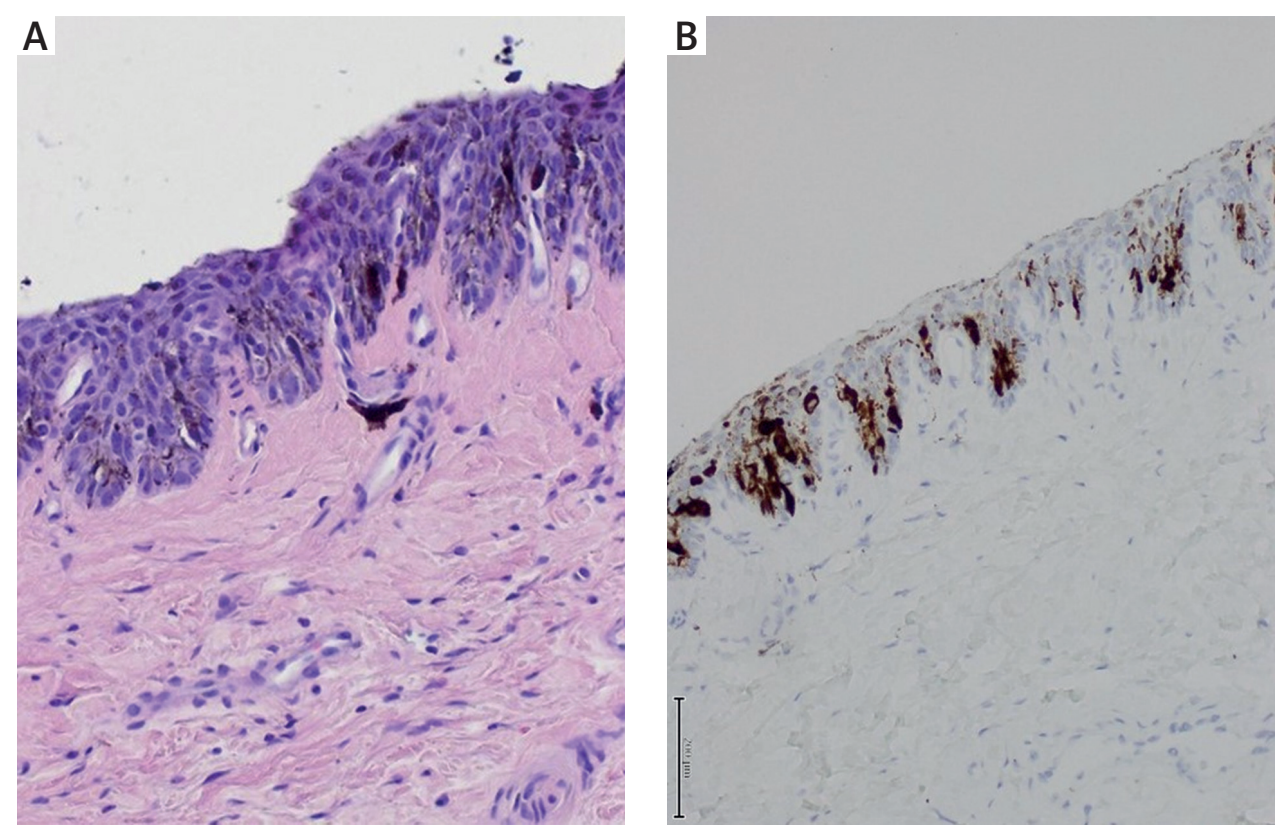

Figure 5. A - Melanocytic proliferation of the lentiginous pattern along the dermoepidermal junction with focal individual epithelioid melanocytes revealing architectural and cytologic atypia (hematoxylin and eosin). B - Melan-A staining of melanocytes at the dermoepidermal junction 


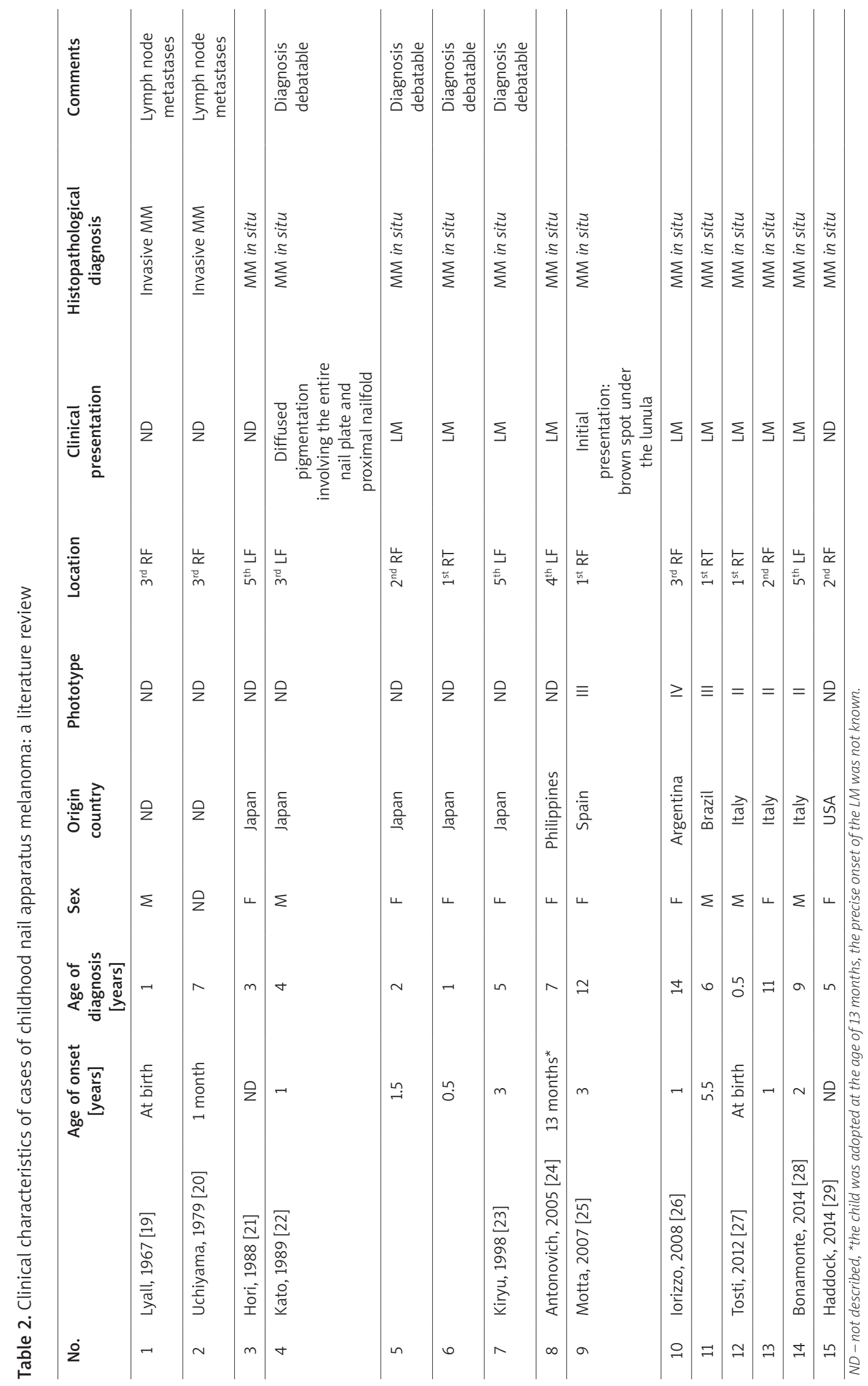


pendently of the studied population, LM in children occurs very rarely. In Chinese research performed by Leung et al. [7], among 461 examined patients aged up to 19 years, no LM cases have been found. In our material, among 348 patients consulted due to various nail apparatus pathologies, 72 cases of LM have been identified, including 8 cases of childhood LM (2.0\%).

The relationship between LM and NAM is currently undeniable. Even though the optimal patient management has not been established yet, most authors recommend histopathological evaluation of the disorder [8, 9]. Unfortunately, it is a painful procedure associated with the risk of permanent nail deformation. Therefore, in childhood LM the biopsy is performed in cases suspected of melanoma or on the parents' request.

The prediction of the course of LM in children is challenging. Although melanoma represents up to $2 \%$ of all diagnosed cancers in children, NAM is very rare. Unfortunately the disease, if occurs, is characterized by high mortality [10].

Goettmann-Bonvallotet al. [11] analysed 40 cases of LM in patients aged less than 16 years. The study identified 19 nevi (17 junctional and 2 compound), 12 lentigines and 9 cases of melanocytes activation as the background of LM. Similarly, Tosti et al. [12] and Léauté-Labrèze et al. [13] did not observe melanoma in 22 and 8 children with LM, respectively. Ohn et al. [14] analysed 58 nail matrix nevi in 56 children and concluded that nail matrix nevi in children significantly more often reveal melanomaassociated features like: multicolour pattern, darker discolouration, pseudo-Hutchinson sign, triangular sign, and the presence of dots/globules.

There are no definite recommendations regarding the management of childhood LM, therefore in our material, dermoscopic indications to biopsy were the same as in adults: the presence of Hutchinson sign, width of the pigmented band $>1 / 3$ of the nail plate width, dark-brown colour of the background and irregular dermoscopic pattern (various colours and thicknesses of the longitudinal lines with uneven intervals) [15]. Another important factor was the change in clinical and dermoscopic presentation, which implicated prompt histopathological evaluation in patient 7.

In the literature some cases of spontaneous regression of childhood LM have been described [16-18]. Murata and Kumano [17] showed that randomly distributed dots and lines that follow the melanocytic lines may be indicators of spontaneous fading of LM in children. In our material, we did not observe these structures in a patient with spontaneous regression.

Our observations and literature data strongly support the rarity of childhood NAM in Caucasian population. To the best of our knowledge, only 15 cases (including 3 that occurred in fair-skinned Caucasians) of NAM in children have been reported so far. Most childhood NAMs described so far presented clinically as LM; the histopathological examination confirmed the diagnosis of melanoma "in situ" (Table 2) [19-29]. Nonetheless, in 2 cases, melanoma had an aggressive course [26]. Interestingly, these both cases did not present as LM, what indicates indirectly that nail matrix melanocytes were not the origin of melanoma.

The difficulties in interpretation of the histopathological picture of nail apparatus pigmentation disorders are being emphasized. Some aspects, considered in adults as evidence of malignancy (e.g. nucleus atypia, moderate migration of melanocytes), may be present in benign pigmented lesions in children, what we present in patient $7[19,20,26,30,31]$. In Goettmann-Bonvallot et al. report [11], nuclear atypia and moderate migration of melanocytes were observed in 15\% and 20\% of cases, respectively. The fact brings into question 4 cases of NAM diagnosed by Kato et al. [22] and Kiryu [23]. Recently, Bonamonte et al. [28] mentioned a possible utility of a novel immunohistochemical marker, anti-p16, in such cases.

\section{Conclusions}

In the light of presented facts, the risk of childhood NAM in Caucasian population appears to be low. The presented cases seem to support the thesis of benign background of LM in children. Histopathological evaluation or removal of the lesion does not seem to be reasonable in every case. Nevertheless, regarding the rarity of this entity (especially in Caucasian population), difficulty in predicting the evolution of the lesion, and the possibility of development of NAM in adults with LM observed since the childhood, regular long-term follow-up should be recommended. Dermoscopy is useful in initial and subsequent patient assessment.

\section{Conflict of interest}

The authors declare no conflict of interest.

\section{References}

1. Sobjanek M, Włodarkiewicz A, Toboła J. Melanonychia longitudinalis. Adv Dermatol Allergol 2006; 23: 130-7.

2. Sobjanek M, Włodarkiewicz A, Urban M, Toboła J. Czerniak aparatu paznokciowego. Dermatol Estet 2007; 1: 18-24.

3. Sobjanek M, Michajłowski I, Włodarkiewicz A, et al. Benign nail apparatus tumours in the material of the Dermatology Venerology and Allergology Department of the Medical University of Gdańsk. Przegl Dermatol 2011; 98: 477-82.

4. Sobjanek M, Michajłowski I, Biernat W, et al. Nail apparatus melanoma: epidemiological, clinical and histopathological analysis. Przegl Dermatol 2011; 98: 384-9.

5. Sobjanek M, Michajłowski I, Konczalska M, et al. Childhood nail alterations in Polish population. Acta Dermatovenerol Croat 2012; 20: 95-7.

6. Haneke E, Baran R. Longitudinal melanonychia. Dermatol Surg 2001; 27: 580-4. 
7. Leung AK, Robson WL, Liu EK, et al. Melanonychia striata in Chinese children and adults. Int I Dermatol 2007; 46: 920-2.

8. Lauteur N, Andre J. Melanonychia: diagnosis and treatment. Derm Therapy 2002; 15: 131-41.

9. Ronger S, Touzet S, Ligeron C, et al. Dermoscopic examination of nail pigmentation. Arch Dermatol 2002; 138: 1327-33.

10. Saenz NC, Saenz-Badillos J, Busam K, et al. Childhood melanoma survival. Cancer 1999; 85: 750-4.

11. Goettmann-Bonvallot S, André J, Belaich S. Longitudinal melanonychia in children: a clinical and histopathologic study of 40 cases. J Am Acad Dermatol 1999; 41: 17-22.

12. Tosti A, Baran R, Piraccini BM, et al. Nail matrix nevi: a clinical and histopathologic study of twenty-two patients. J Am Acad Dermatol 1999; 41: 17-22.

13. Léauté-Labrèze C, Bioulac-Sage P, Taïeb A. Longitudinal melanonychia in children. A study of eight cases. Arch Dermatol 1996; 132: 167-9.

14. Ohn J, Choe YS, Mun JH. Dermoscopic features of nail matrix nevus (NMN) in adults and children: a comparative analysis. J Am Acad Dermatol 2016; 75: 535-40.

15. Sobjanek M, Michajłowski I, Biernat W, et al. Clinical and dermoscopic features of nail apparatus pigmentations. Przegl Dermatol 2013; 100: 65-79.

16. Tosti A, Baran R, Morelli R, et al. Progressive fading of longitudinal melanonychia due to a nail matrix melanocytic nevus in a child. Arch Dermatol 1994; 130: 1076-7.

17. Murata Y, Kumano K. Dots and lines: a dermoscopic sign of regression of longitudinal melanonychia in children. Cutis 2012; 90: 293-6.

18. Kikuchi I, Inoue S, Sakaguchi E, Ono T. Regressing nevoid melanosis in childhood. Dermatology 1993; 186: 88-93.

19. Lyall D. Malignant melanoma in infancy. J Am Med Assoc 1967; 202: 93.

20. Uchiyama M, Minemura K. Two cases of malignant melanoma in young persons. Nippon Hifuka Gakkai Zasshi 1979; 89: 668.

21. Hori Y, Yamada A, Takematsu U, et al. Pigmented small tumors. Jpn J Pediatr Dermatol 1988; 7: 117-20.

22. Kato T, Usuba Y, Takematsu H, et al. A rapidly growing pigmented nail streak resulting in diffuse melanosis of the nail. A possible sign of subungual melanoma in situ. Cancer 1989; 64: 2191-8

23. Kiryu H. Malignant melanoma in situ arising in the nail unit of a child. J Dermatol 1998; 25: 41-4.

24. Antonovich DD, Grin C, Grant-Kels JM. Childhood subungual melanoma in situ in diffuse nail melanosis beginning as expanding longitudinal melanonychia. Ped Dermatol 2005; 22: 210-2.

25. Motta A, López C, Acosta A, et al. Subungual melanoma in situ in a Hispanic girl treated with functional resection and reconstruction with onychocutaneous toe free flap. Arch Dermatol 2007; 143: 1600-2.

26. Iorizzo M, Tosti A, Di Chiacchio N, et al. Nail melanoma in children: differential diagnosis and management. Dermatol Surg 2008; 34: 974-8.

27. Tosti A, Piraccini BM, Cagalli A, Haneke E. In situ melanoma of the nail unit in children: report of two cases in fair-skinned Caucasian children. Pediatr Dermatol 2012; 29: 79-83.

28. Bonamonte D, Arpaia N, Cimmino A, Vestita M. In situ melanoma of the nail unit presenting as a rapid growing longitudinal melanonychia in a 9-year-old white boy. Dermatol Surg 2014; 40: 1154-7.

29. Haddock NT, Wilson SC, Shapiro RL, Choi M. Wide local en bloc excision of subungual melanoma in situ. Ann Plast Surg 2014; 73: 640-4.
30. Evans MJ, Gray ES, Blessing K. Histopathological features of acral melanocytic nevi in children: study of 21 cases. Pediatr Dev Pathol 1998; 1: 388092.

31. Elder DE. Precursors to melanoma and their mimics: nevi of special sites. Mod Pathol 2006; 19: S4-20. 